\title{
A COMPARISON OF SINGLE PHOTON AND FULL WAVEFORM LIDAR
}

\author{
G. Mandlburger ${ }^{1,2 *}$, H. Lehner ${ }^{3}$, N. Pfeifer ${ }^{1}$ \\ ${ }^{1}$ TU Wien, Department of Geodesy and Geoinformation, Austria - [gottfried.mandlburger, norbert.pfeifer]@geo.tuwien.ac.at \\ ${ }^{2}$ University of Stuttgart, Institute for Photogrammetry, Germany - gottfried.mandlburger@ifp.uni-stuttgart.de \\ ${ }^{3}$ City of Vienna, Municipal Department 41, Austria - hubert.lehner@wien.gv.at
}

\author{
Commission II, WG II/2
}

KEY WORDS: Laser Scanning, Light Detection and Ranging, Single Photon Sensitivity, Vegetation Penetration, 3D City Mapping

\begin{abstract}
:
Single photon sensitive LiDAR sensors are currently competing with conventional multi-photon laser scanning systems. The advantage of the prior is the potentially higher area coverage performance, which comes at the price of an increased outlier rate and a lower ranging accuracy. In this contribution, the principles of both technologies are reviewed with special emphasis on their respective properties. In addition, a comparison of Single Photon LiDAR (SPL) and Full Waveform LiDAR data acquired in July and September 2018 in the City of Vienna are presented. From data analysis we concluded that (i) less flight strips are needed to cover the same area with comparable point density with SPL, (ii) the sharpness of the resulting 3D point cloud is higher for the waveform LiDAR dataset, (iii) SPL exhibits moderate vegetation penetration under leaf-on conditions, and (iv) the dispersion of the SPL point cloud assessed in smooth horizontal surface parts competes with waveform LiDAR but is higher by a factor of 2-3 for inclined and grassy surfaces, respectively. Still, SPL yielded satisfactory precision measures mostly below $10 \mathrm{~cm}$.
\end{abstract}

\section{INTRODUCTION}

In the recent past, the advent of single photon sensitive detection has gained attention in the airborne LiDAR (Light Detection And Ranging) sector constituting a potential alternative to conventional Full Waveform (FWF) based sensors. Two systems became specifically important in the commercial sector: The Geiger-mode LiDAR (GmLiDAR) system operated as a service by Harris Corporation (Harris, 2019) in the U.S. and Single Photon LiDAR, which recently became commercially available under the brand SPL100 (Leica, 2019) with the acquisition of the sensor manufacturer Sigma Space Corporation by Hexagon/Leica Geosystems.

Both GmLiDAR and SPL use low energy laser pulses and focal plane array detectors (Stoker et al., 2016), i.e., the reflected optical power of a single pulse is captured my multiple detectors. In contrast to GmLiDAR, SPL features multi-target capability, which is especially important for penetrating semi-transparent targets like vegetation. Due to the high receiver sensitivity, both systems can be operated from high altitudes of approx. 4000$10000 \mathrm{~m}$ above ground level (AGL) and thus enable a higher area performance compared to conventional laser scanners while providing similar point densities $\left(800-1000 \mathrm{~km}^{2} / \mathrm{h}\right.$ with 8 points $/ \mathrm{m}^{2}$, Leica (2019)) at the prize of a lower height accuracy $(10 \mathrm{~cm})$ and a higher rate of outlier points (Ullrich and Pfennigbauer, 2018).

The higher area performance is particularly beneficial for countrywide topographic mapping. Both GmLiDAR and SPL are therefore deployed within the 3 D Elevation Program (Sugarbaker et al., 2014), an initiative for the acquisition of nationwide elevation data in the U.S., and both technologies are evaluated with respect to the adherence to the requested quality standards (Stoker et al., 2016). To date, one SPL100 sensor is operated in Europe and the sensor is tested in several pilot projects (e.g. Finland, The Netherlands, Spain, Germany, Austria). Capturing of

\footnotetext{
${ }^{*}$ Corresponding author
}

the province Navarra in Northern Spain is an example for the use of the SPL100 in a production environment. The respective data were recently published as open data* . In Austria, among others the municipal administration of the City of Vienna has commissioned a flight for testing the capabilities of Single Photon LiDAR especially for urban mapping. The same area has also been captured with a conventional FWF laser scanning system in the same season.

The aim of this paper is to first briefly introduce the functional principles of both Single Photon and Full Waveform LiDAR focusing on the technological differences of the respective techniques (Section 2). After describing the technologies, we present an experiment comparing SPL and FWF LiDAR based on datasets of the City of Vienna captured in July/September 2018 with a Leica SPL100 and a Riegl VQ-1560i. Section 3 details the study area and the processing methods used for data evaluation. First results of this case study are presented and discussed in Section 4. The final Section 5 summarizes the main findings and provides an outlook on future research activities.

\section{PRINCIPLES OF FUNCTION}

\subsection{Basics}

Airborne Laser Scanning (ALS), or airborne LiDAR respectively, is a dynamic, polar, and active multi-sensor 3D data acquisition technique comprising a navigation unit (GNSS, IMU) determining of the position and attitude of the sensor platform and the laser scanner. The latter provides the scan angle of the laser beam and the distance between the sensor and the reflecting targets (Shan and Toth, 2018; Vosselman and Maas, 2010). Distances are estimated by measuring the round trip time of a short laser pulse.

\footnotetext{
*ftp://ftp.cartografia.navarra.es/5_LIDAR/5_4_2017_ NAV_cam_EPSG25830/
} 

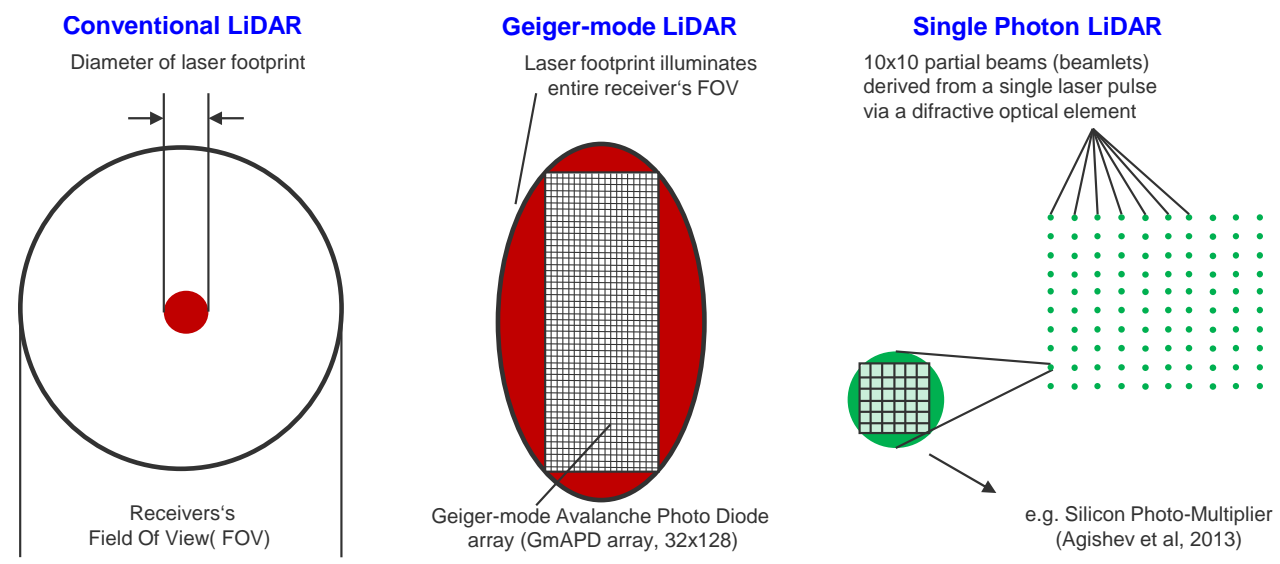

Figure 1. Principle sketch: Incoming laser radiation from the receiver's perspective

If the propagation speed is known, the range can be estimated from the time-of-flight of the laser pulse. Furthermore, a deflection unit (oscillating, rotating or nutating mirror) together with the forward motion of the aircraft provides systematical scanning of the topography.

This general principle applies to both conventional and single photon sensitive laser scanning. The main difference is the employed detector technology which is detailed in the following subsection. Figure 1 sketches the basic situation of the incoming laser radiation seen from the receiver's perspective.

\subsection{Linear-mode Full Waveform LiDAR}

Conventional time-of-flight laser ranging, also referred to as linear-mode LiDAR in contrast to single photon sensitive Geigermode LiDAR, uses a highly collimated laser pulse whose backscattered signal is received by a single detector. The receiver's Field of View (FoV) is typically considerably larger than the diameter of the reflected laser beam (cf. Figure 1a). For the conversion of the optical power into digital radiometric information, a two-stage procedure is employed (Ullrich and Pfennigbauer, 2016): First, an Avalanche Photo Diode (APD) converts the received laser radiation into an analog signal, and subsequently an Analog Digital Converter (ADC) generates the final measurement in digital form. The term linear-mode indicates the dynamic range of the APD where the optical power and the ana$\log$ output are linearly related. For the sake of completeness, it is noted that modern sensors employ multiple APDs/ADCs with different sensitivity to increase the achievable dynamic range. APDs operated in linear mode deliver measures of the received signal strength and provide object reflectance and/or material properties of the illuminated objects via radiometric calibration (Wagner, 2010; Lehner and Briese, 2010; Briese et al., 2012).

The actual range detection is either implemented by hardware components of the laser scanner (discrete echo systems) or by high-frequency discretization of the entire backscattered echo waveform. The captured waveforms are either processed online by the firmware of the sensor (Pfennigbauer et al., 2014) or stored for detailed analysis in postprocessing (Shan and Toth, 2018). To date, full waveform acquisition is only feasible for conventional LiDAR systems with entailed advantages w.r.t. ranging precision, target separability and object characterization (echo width, slope, reflectance). However, at least several hundred photons are required for a reliable detection of a single object, and predominantly infrared wavelengths $(\lambda=1024 / 1550 \mathrm{~nm})$ are employed for topographic applications. Even very sensitive single detector systems used for airborne laser bathymetry, operating with laser radiation in the visible green domain of the spectrum $(\lambda=532 \mathrm{~nm})$, require about 250 photons for successful echo detection (Mandlburger and Jutzi, 2018). Apart from the flight velocity and altitude, the scan mechanism, and the reflectance of the illuminated objects, the achievable point density mainly depends on the laser pulse repetition rate.

\subsection{Geiger-mode LiDAR}

In GmLiDAR, a divergent laser pulse is emitted resulting in a large laser footprint on the ground. The reflected signal is captured by a Geiger-mode Avalanche Photo Diode (GmAPD) array, i.e., a matrix of single photon sensitive receiver elements (Kim et al., 2013; Degnan, 2016; Stoker et al., 2016). The APD of each single matrix element is operated in Geiger-mode, where an additional bias above the break-through voltage brings the detector in a state where the arrival of a single or a few photons is sufficient to trigger the avalanche effect leading to an abrupt rise of voltage at the detector's output (Ullrich and Pfennigbauer, 2016). Each cell is therefore a binary detector, and the break-through event of the photodiode triggers the stop impulse for the range estimation via a Time-To-Digital convert (TDC). After a break-through event, the respective cell is inactive for a longer period of time. Thus, after the detection of a first echo, no further echo can be measured from the same laser pulse. Only after a reset of the entire GmAPD array in the course of the emission of a subsequent laser pulse, all cells are active again.

Because of the single photon sensitivity, much higher flying altitudes are feasible (Clifton et al., 2015) potentially increasing the area performance. The resulting point density decrease is compensated by the detector array, which can be seen as a range camera that spatially sub-samples the large footprint area. The detector's FoV is large enough to illuminate all $32 \times 128=1024$ detector cells (cf. Figure 1b). In contrast to conventional LiDAR, the spatial resolution of GmLiDAR is thus limited by the size of the detector cells (and the flying altitude) rather than by the size of the laser footprint on the ground. The system operated by Harris Corporation, for instance, features a cell size of $35 \mu \mathrm{m}$ resulting in a point spacing on the ground of $35 \mathrm{~cm}$ at a flying altitude of $10000 \mathrm{~m}$ AGL. Such a small point spacing is only achieved if all echoes stem from reflections at actual targets (ground, buildings, vegetation, infrastructure...). In practice, reflections at aerosol particles also trigger echo events, but the point density further increases as consecutive laser footprints are highly overlapping (Romano, 2015). In open terrain, the high point density can be 
used to smooth the higher ranging errors. Vegetation penetration, in turn, is only moderate due to the technology's lack of multi-target capability. Finally, it is noted that the Harris system is optimized for efficient large-area topography acquisition and utilizes an infrared laser $(\lambda=1024 \mathrm{~nm})$. Due to the binary character of the detector, radiometric information cannot be measured directly but is rather estimated from the local point density.

\subsection{Single Photon LiDAR}

In contrast to that, the technology referred to as Single Photon LiDAR (SPL) utilizes a very short laser pulse (pulse duration: $400 \mathrm{ps}$ ), which is split into a grid $10 \times 10$ sub-beams (beamlets) by a diffractive optical element (DOE) (Degnan, 2016; Leica, 2019). The 100 beamlets are highly collimated (nominal beam divergence at $\left.1 / e^{2}: 0.08 \mathrm{mrad}\right)$, thus their footprints on the ground do not overlap (cf. Figure 1c). For each beamlet, the backscattered signal is received by an individual detector which is aligned to the laser beam direction. Each detector, in turn, consists of a matrix of several hundred single photon sensitive cells, and each cell is operated in Geiger-mode. The exact technical realisation is not disclosed by the manufacturer. Possible implementations include Micro Channel Plate PhotoMultiplier Tubes (MCP-PMT) or Silicon PhotoMultipliers (SiPM) (Agishev et al., 2013; Degnan, 2002).

As described above, the individual SPL beamlet detectors are technically cell arrays but, in contrast to GmAPD arrays, they do not feature further spatial subsampling but rather provide a cumulative signal at the detecor's output. As some elements of the micro-cell array may respond to targets closer to the sensor (e.g. vegetation) while others trigger later at more distant targets (e.g. ground), this technology has inherent multi-target capabilities. In fact, in a certain dynamic range each beamlet detector acts like an Avalanche Photo Diode (APD) operated in linear-mode. To date, temporal discretization of the signal and storage of the echo waveform is not feasible. However, in contrast to $\mathrm{GmLi}$ DAR, the cumulative signal intensity at the detector's output can be measured but it's radiometric resolution is less compared to conventional multi-photon based APDs.

The SPL100 is a hybrid multi-sensor system comprising an RCD30 80 Mpix RGBI camera along with the laser scanner and the mandatory navigation unit (Leica, 2019). The sensor is optimized for efficient acquisition of extensive topography, but is also suitable for mapping shallow water bathymetry (Degnan, 2016) due to the employed laser wavelength in the visible green $(\lambda=532 \mathrm{~nm})$, the conical scan mechanism (Palmer scanner) with selectable off-nadir angles of $10^{\circ}, 15^{\circ}, 20^{\circ}$, or $30^{\circ}$, and the high detector sensitivity.

As for GmLiDAR, the single photon sensitivity of SPL enables higher flying altitudes and consequently a potentially higher area performance compared to conventional LiDAR. As stated above, this is especially relevant for nationwide topographic mapping. Due to (i) the general availability of the system outside the U.S. and (ii) the inherent multi-target capability, an inevitable precondition for capturing large forested areas, the remaining part of the manuscript focuses on a comparison of SPL and FWF laser scanning. The latter is subsequently referred to as waveform LiDAR.

\section{MATERIALS AND METHODS}

\subsection{Study area and datasets}

To evaluate the SPL technology for urban mapping, the surveying department of the municipality of the City of Vienna (MA41) commissioned company COWI (Denmark) with the acquisition of a $160 \mathrm{~km}^{2}$ test area, roughly one third of the area of the city. The captured flight block (length: $20 \mathrm{~km}$, width: $8 \mathrm{~km}$ ) stretches from the Lobau area east of the Danube river, via the city center to the adjoining forested area in the western part of city (cf. Figure 2a). Data acquisition was carried out on July 29, 2018 with the Hexagon/Leica Geosystems SPL100 sensor flown at an altitude of $4000 \mathrm{~m}$ with a pulse repetition rate (PRR) of $50 \mathrm{kHz}$ resulting in an effective scan rate of $5 \mathrm{MHz}$. The product specification requested a last echo point density of 20 points $/ \mathrm{m}^{2}$. To achieve good area performance, a $15^{\circ}$ scanning wedge was employed resulting in a swath width of approx. $2000 \mathrm{~m}$. In order to minimize scan shadows in the built-up area, the flight was planned with approx. $60 \%$ side overlap. This resulted in 10 flight strips, while only 5 strips would have been necessary to cover the entire area with $20 \%$ overlap.

The same area was captured with a conventional FWF laser scanner (Riegl VQ-1560i) on September 20, 2018, thus, at a comparable state of foliation (leaf-on). Figure $2 \mathrm{~b}$ depicts the entire flight block, which was planned to (i) satisfy the same single strip point density as mentioned above and (ii) to maintain sufficient coverage at street level even in urban canyon areas. The entire block consists of 18 flight strips with a side overlap $>50 \%$ flown at an altitude of $750 \mathrm{~m}$ AGL with a speed of 120 knots $(60 \mathrm{~m} / \mathrm{s})$ and an effective scan rate of $1.33 \mathrm{MHz}$. The scanner employs two independent laser sources. The pulses of these lasers are deflected via the same rotating prim, forming $\mathrm{X}$-shaped scan lines on the ground which are rotated by $\pm 14^{\circ}$ against the flight direction's perpendicular axis. Each of the two vertical scan planes features a scan angle range of $\pm 30^{\circ}$ resulting in a total lateral FoV of $58^{\circ}$. The swath width therefore amounts to $840 \mathrm{~m}$. The instrument uses online waveform processing for target (echo) detection and characterization, and provides additional attributes like signal amplitude, target reflectance, and a quality measure (pulse shape deviation) for each echo next to the $3 \mathrm{D}$ position.

From the entire flight block two representative $400 \times 400 \mathrm{~m}^{2}$ areas were extracted and analyzed in detail (cf. Figure 3 ). The respective locations are marked with a red rectangle in Figure 2a. The eastern patch represents a typical urban area and features multistory building blocks, street canyons, and a church with a $70 \mathrm{~m}$ high tower (cf. Figure 3b). This dataset allows for a comparison of the coverage within narrow street canyons and it enables assessment of the measurement precision at smooth surfaces (roofs, streets, etc.). The western section constitutes a forested area with stands of different height, forest roads, and an open area with a free standing building (cf. Figure 3a). This patch is suitable for evaluation the penetration capabilities of SPL and waveform LiDAR, respectively. The study areas are referred to as "city" and "forest" in the following.

\subsection{Data processing methods}

The following processing steps have been applied to both test areas (city, forest) and both data acquisitions (SPL100, VQ-1560i):

- Visual inspection in a 3D point cloud viewer: For the SPL100 dataset, this analysis is performed based on the un- 

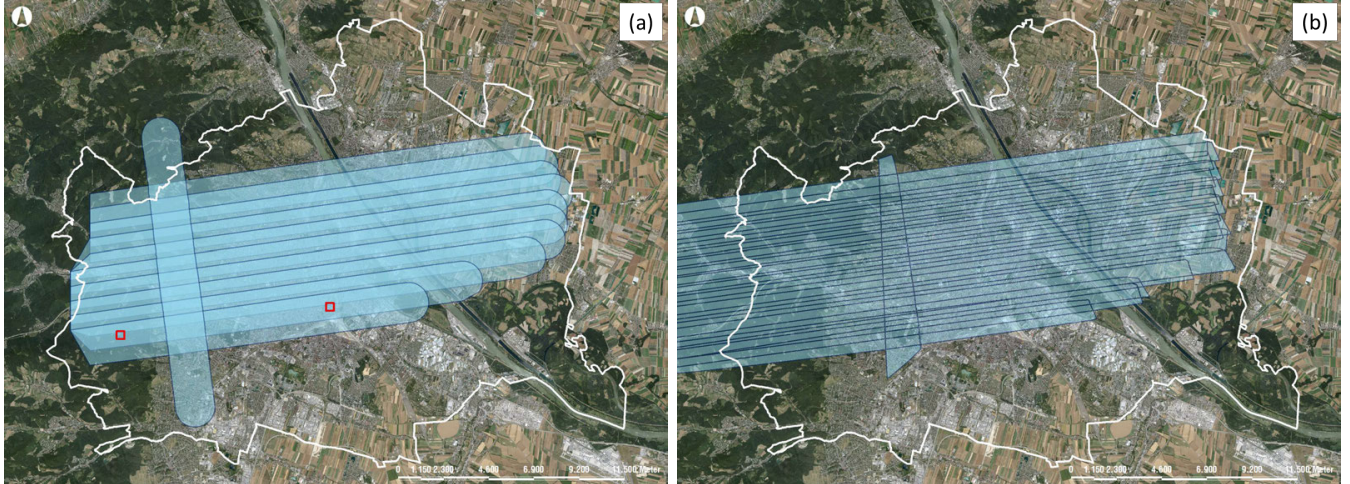

Figure 2. Flight block Vienna; (a) Single Photon LiDAR, (b) waveform LiDAR, red rectangles: detailed test areas (cf. Figure 3)
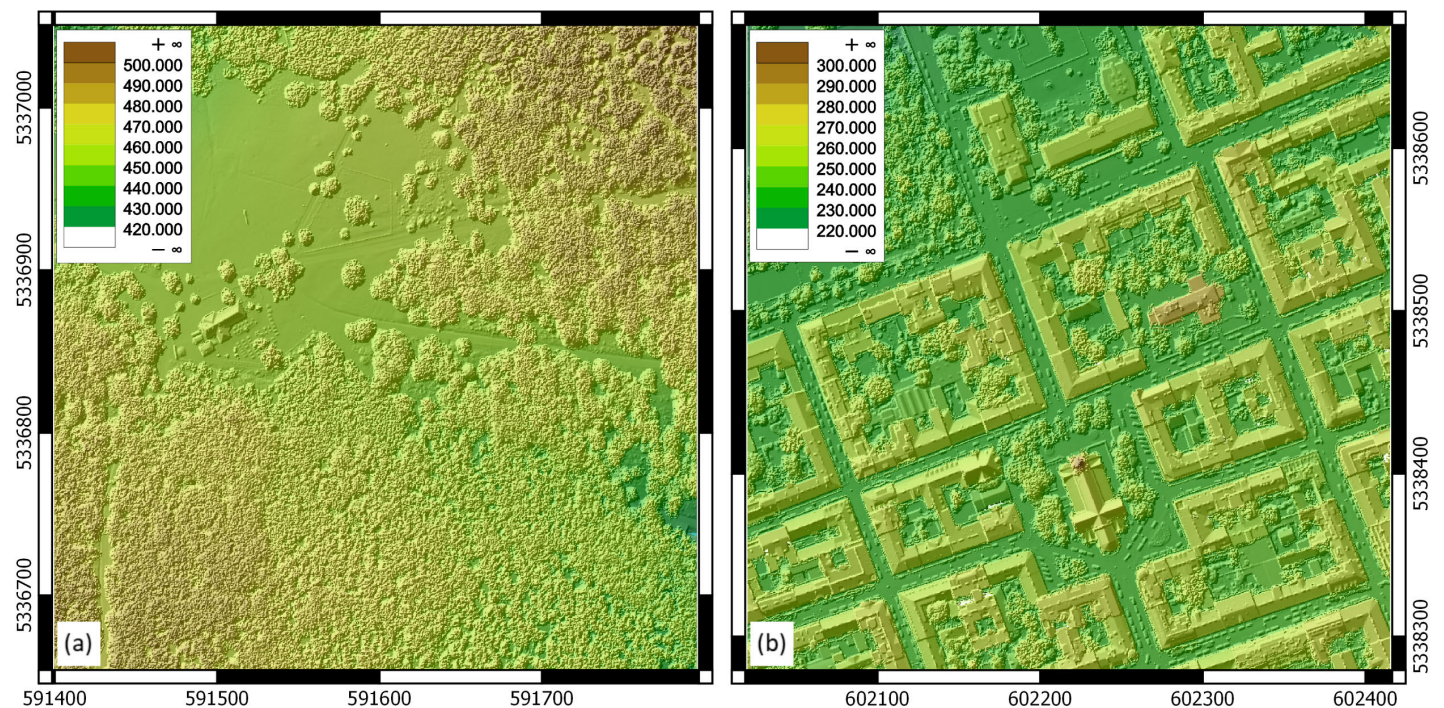

Figure 3. Shaded relief map of test areas superimposed with color coded height map, (a) forest, (b) city

filtered point cloud including clutter points above and below the terrain.

- Penetration rate: The assessment is carried by visual inspection based on sections and by analyzing the return number statistics.

- Point density: Quantitative evaluation by sorting the laser echoes into $2.5 \mathrm{~m}$ cells and by visualizing and statistically analyzing the resulting raster models.

- Strip height differences: Interpolation of strip-wise Digital Elevation Models (DEM), classification of smooth areas based on the standard deviation of the grid post's elevation (interpolation: moving tilted planes), and calculation of DEM of Difference models for overlapping flight strips.

- Assessment of measurement noise: In smooth areas, the interpolation error (std.dev.) is a precision indicator. The residual deviations of the data points w.r.t. their best fitting tilted plane characterize the measurement precision. The quantitative assessment is carried out by statistical analysis (histograms, statistical parameters).

\section{RESULTS AND DISCUSSIONS}

In this section, the data evaluation results of the Single Photon (SPL100) and waveform LiDAR (VQ-1560i) datasets are presented and discussed. In contrast to existing studies (Swatantran et al., 2016), which compare up-to-date SPL data acquisitions with conventional LiDAR data of lower resolution, we assess two datasets which (i) both feature state-of-the art sensors of the respective technology, and (ii) are the result of data acquisitions with the same underlying specification in terms of point density (20 points $/ \mathrm{m}^{2}$ and side overlap $50 \%$ ).

Due to the single photon sensitivity, the SPL technology enables higher flying altitudes resulting in larger swath widths compared to conventional LiDAR. The flight block overview (Figure 2) shows that only 10 flight strips are necessary to cover the entire project area with an effective strip overlap of $65 \%$ using the SPL system compared to 18 flight lines for the waveform LiDAR sensor. Thus, the SPL flight lines are more than twice as wide as the waveform LiDAR strips ( $2400 \mathrm{~m}$ vs. $840 \mathrm{~m}$ ). In order to meet the requested point density of 20 points $/ \mathrm{m}^{2}$ while flying 5 times higher than the conventional laser scanner $(4000 \mathrm{~m}$ vs. $750 \mathrm{~m})$, the SPL sensor uses a higher effective scan rate of $5 \mathrm{MHz}$ (pulse repetition rate: $50 \mathrm{kHz}, 100$ beamlets).

Due to the high detector sensitivity, weak reflections at aerosol particles in the atmosphere trigger false echoes. One of the major processing steps within the SPL data processing pipeline is therefore the separation of real target points (ground, vegetation, buildings, etc.) and clutter points. A representative example is depicted in Figure 4 showing a building block of the city test area in a perspective view (Figure 4a) and a section view thereof (Figure $4 \mathrm{~b}$ ), both colored by intensity. It can be seen that the clutter 
(a)

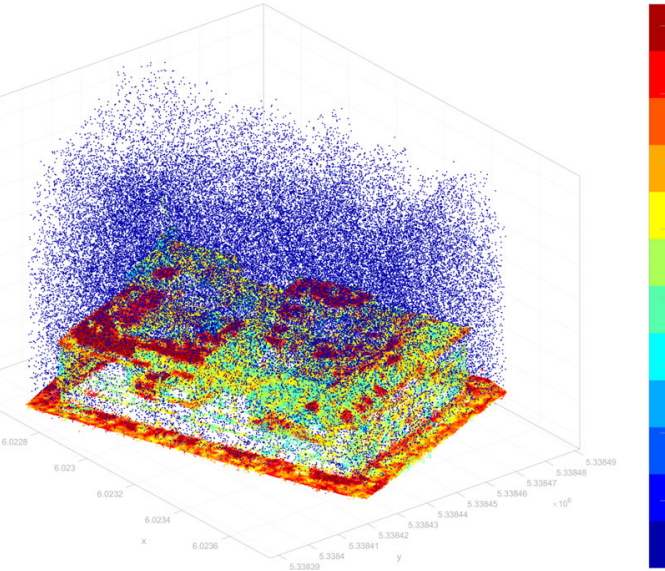

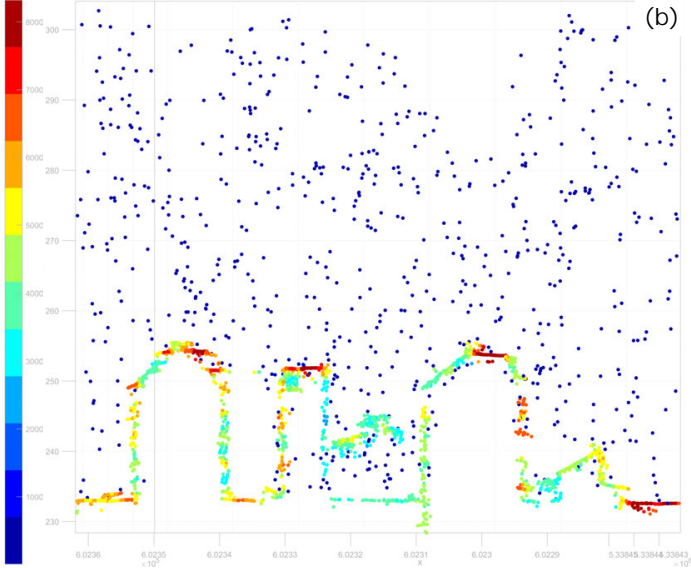

Figure 4. Unfiltered SPL100 3D point cloud of a building block within the city test area colored by intensity [DN]; (a) perspective view of the entire building block, (b) section view of a subarea

(a)

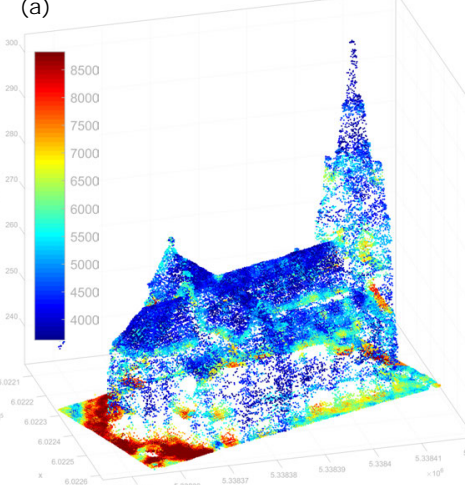

(d)

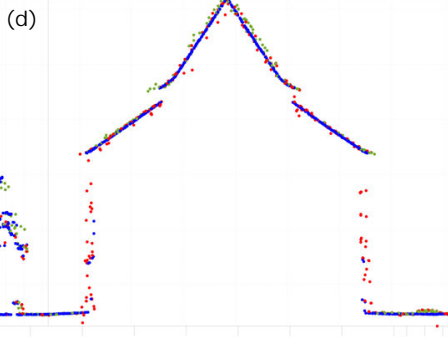

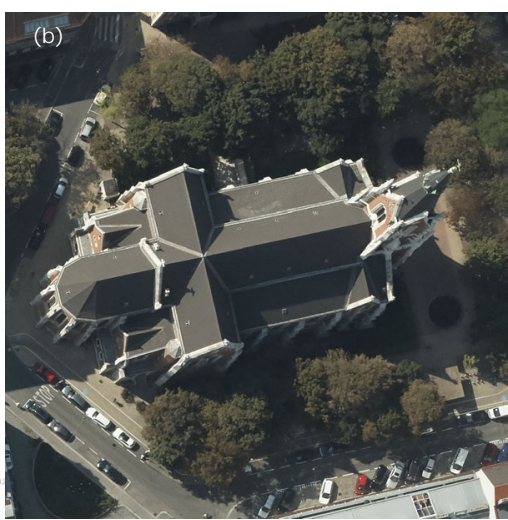

(e)

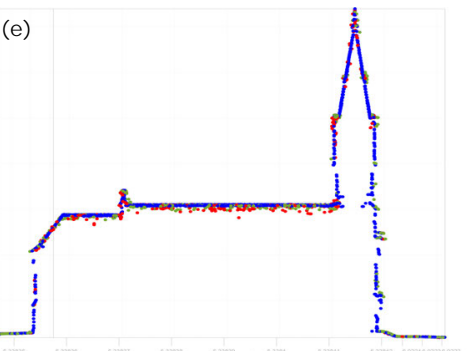

(c)

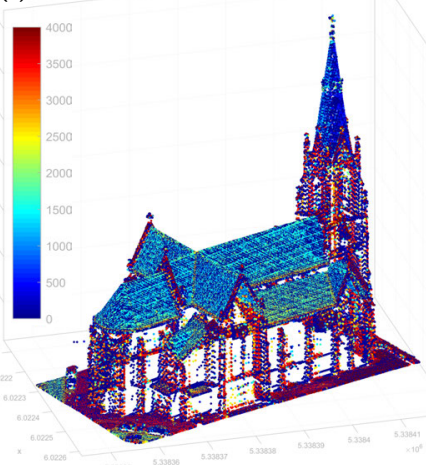

(f)

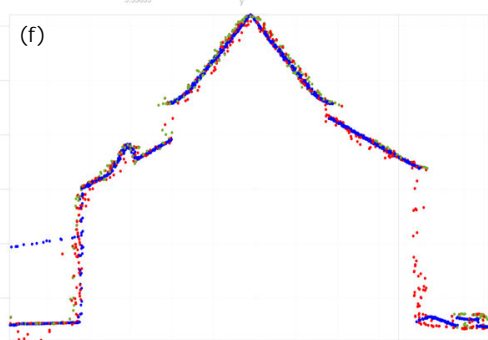

Figure 5. 3D point cloud of the church (city test area) after clutter point removal; (a) perspective view of SPL point cloud colored by intensity [DN], (b) aerial image (PhaseOne, September 2018), (c) perspective view of waveform LiDAR point cloud colored by signal amplitude [DN], (d-f) section views (cross, longitudinal, diagonal): SPL unfiltered/postprocessed (red/green), waveform LiDAR (blue)

points mainly exhibit low intensity values and their 3D distribution is sparse. These two properties are key for SPL point cloud filtering (Degnan, 2016). In contrast, filtering of high-intensity points just below the surface (cf. leftmost and rightmost part of Figure 4b) is demanding, as most well established terrain filtering methods give preference to low lying points.

Figure 5 shows an aerial image (b) and the 3D point clouds (a,cf) of a church located in the southern part of the city test area. In contrast to Figure 4, the clutter points have been removed for both the SPL and the waveform LiDAR dataset. Figure 5 clearly reveals a greater sharpness of the waveform LiDAR points (c) compared to SPL (a), which are both colorized by signal strength. Architectural details, e.g. the church tower, are well captured in the waveform LiDAR point cloud and appear more fuzzy in the SPL data. Moreover, the intensity-based colors of the waveform LiDAR point cloud are clearly related to material properties when comparing them to the aerial image. The relationship is less ob- vious for the SPL points.

Each of the three transects displayed in the lower row of Figure 5 comprises points of the following data sources: (red) SPL freed from clutter points, but otherwise unfiltered, (green) SPL postprocessed, and (blue) waveform LiDAR. While all datasets show a good agreement on the ground surrounding the church, deviations between the point clouds are visible within the steep roof areas. The dispersion of the SPL points is generally higher compared to the waveform LiDAR data. This applies to both the postprocessed (green) and unfiltered (red) dataset. It is noted that the unfiltered points were only available for a single strip, while the other two datasets comprise the points of two overlapping flight strips. However, by comparing the two SPL variants it became obvious, that the process of filtering the raw SPL point cloud also modifies the points. The postprocessed (green) points appear smoother by tendency. Another striking detail of the section views is that a few SPL points are located beyond the roof 
(a)

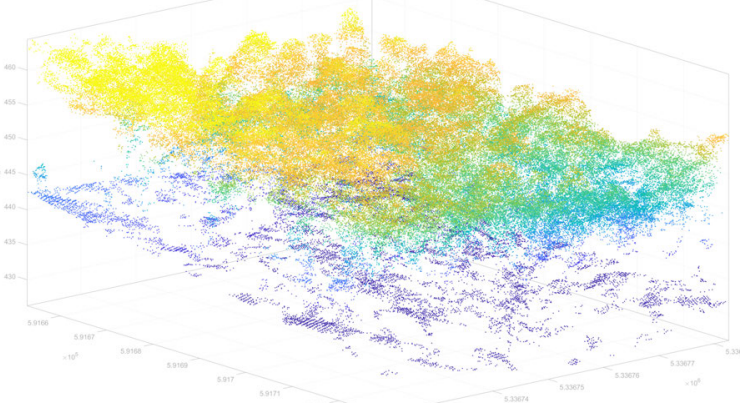

(b)

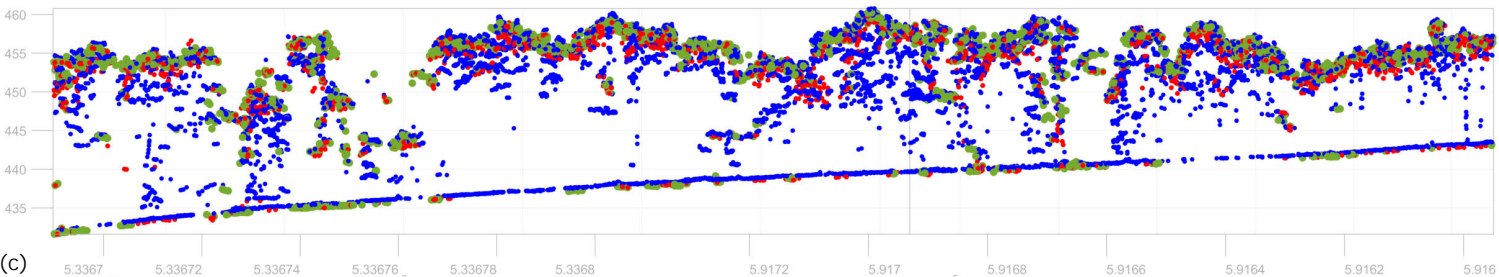

Figure 6. 3D point cloud of forest test area; (a) SPL, (b) Waveform LiDAR, (c) longitudinal section: SPL unfiltered/postprocessed (red/green), waveform LiDAR (blue)
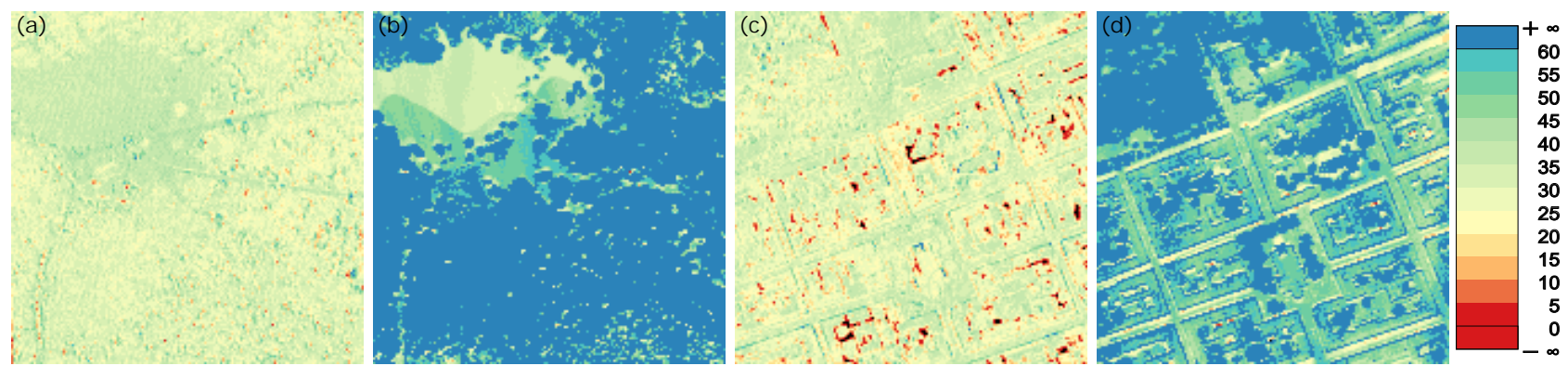

Figure 7. Color coded point density maps [points $/ \mathrm{m}^{2}$ ]; (a/b) forest: SPL/waveform LiDAR, (c/d) city: SPL/waveform LiDAR

perimeter as captured by the waveform LiDAR points. This is rather unexpected as the nominal laser footprint diameter of the SPL100 sensor only amounts to $32 \mathrm{~cm}$ due to the small beam divergence of $0.08 \mathrm{mrad}$ (Leica, 2019). Fuzzy behavior at sharp edges, in turn, is rather observed for scanners with a larger beam divergence as, e.g., used in laser bathymetry due to eye safety considerations.

To assess the vegetation penetration capabilities of SPL, a densely vegetated section of the forest test area is depicted in Figure 6. The upper row shows the SPL (a) and waveform LiDAR (b) point cloud, respectively, in the same perspective viewing geometry. It is apparent that the waveform LiDAR dataset outperforms SPL in terms of ground coverage and overall point density while both datasets were acquired under full leaf-on conditions. The section view of Figure $6 \mathrm{c}$ also supports this statement and, in addition, reveals that waveform LiDAR also provides echoes from within the vegetation next to the canopy and ground. Especially the postprocessed SPL data points are mainly aggregated in the canopy and only a few laser shots reach the ground beneath. For the depicted area, the waveform LiDAR system delivers up to six returns per laser pulse and the mean number of returns amounts to 1.84 compared to 1.06 for the postprocessed SPL points. However, the unfiltered SPL points feature more multi-target echoes with up to five returns per pulse. Thus, postprocessing of the point cloud carried out by the SPL service provider might have eliminated usable points in the vegetation.
Figure 7 depicts color coded point density maps for both test areas and acquisition methods. In order to further study the multi-target and penetration capabilities, all laser returns were used instead of last echoes only. Figure $7 \mathrm{a}$ and $\mathrm{b}$ show the results for the forest test area, which support the statements above. While the open area in the center of the scene stands out in the waveform LiDAR dataset with greenish color tones indicating a point density of approx. 30 points $/ \mathrm{m}^{2}$ compared to the dominating blue colors in the surrounding forest area with a density above 60 points $/ \mathrm{m}^{2}$, the SPL point density is even lower in the vegetated area compared to the open space. This underlines the higher penetration rate of the waveform LiDAR dataset.

The point density maps of the city test area (Figure $7 \mathrm{c}$ and d) on the one hand reveal a generally lower SPL point density. The mean last echo point density (not shown in Figure 7) amounts to 15 points $/ \mathrm{m}^{2}$ for the SPL dataset and $>20$ points $/ \mathrm{m}^{2}$ for the waveform LiDAR data. The overall bias is not related to technological differences but rather a matter of flight planning, and is therefore not discussed further here. However, the relative color patterns allow the following interpretation: In the waveform LiDAR dataset (Figure 7d), the point density between the building blocks often shows a typical sequence of mean density at the roofs (green), followed by high density (blue) at the building edge, a low-density section (yellow) near the houses, and again mean point density (green) in the middle of the street. The density drop in the surrounding of the buildings can be attributed to scan shadows, so that certain areas in the vicinity of buildings are only seen from 

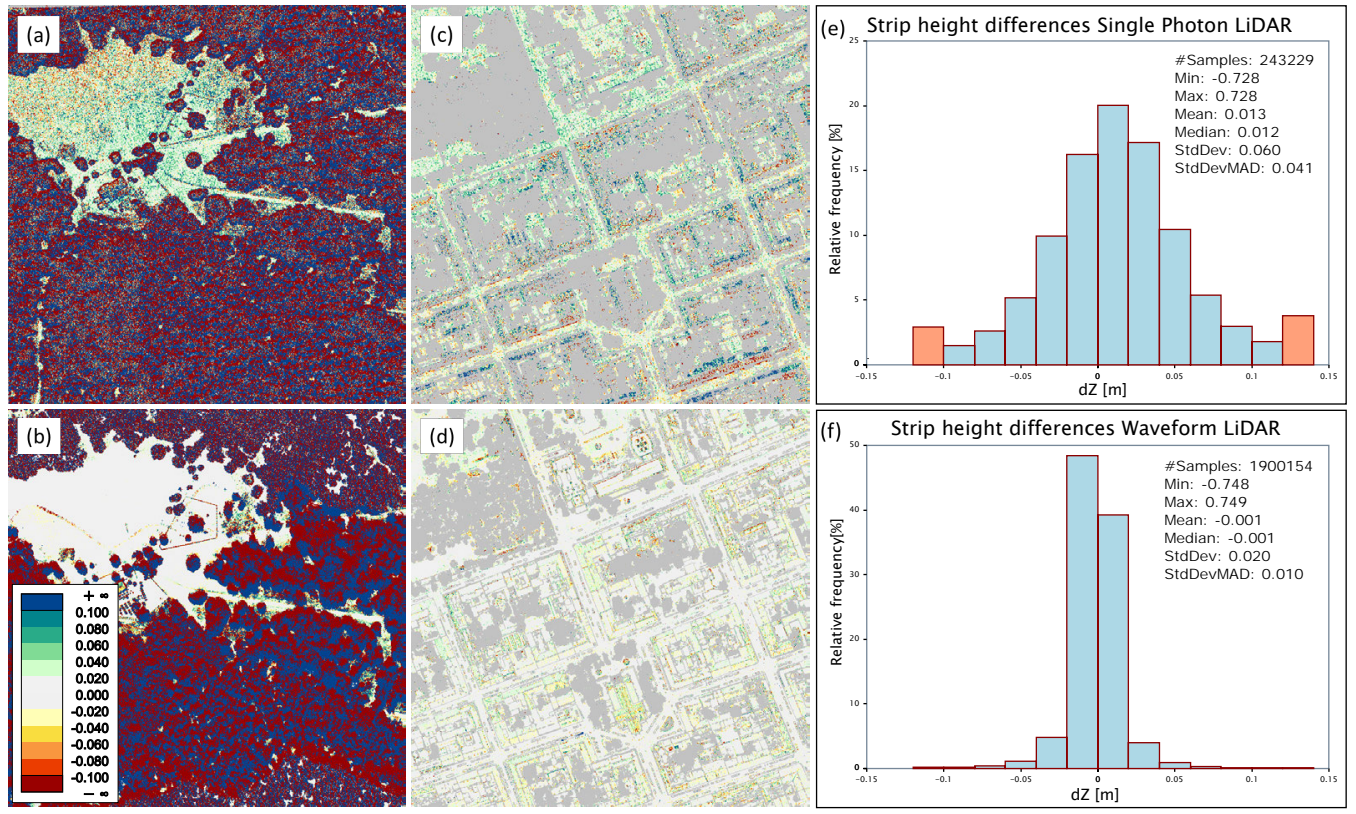

Figure 8. Strip height differences [m]: upper row: SPL, lower row: Waveform LiDAR; $(a+b)$ forest test area unmasked, (c+d) city test area, smooth surface parts only, $(\mathrm{e}+\mathrm{f})$ Histograms of strip differences corresponding to $(\mathrm{c}+\mathrm{d})$
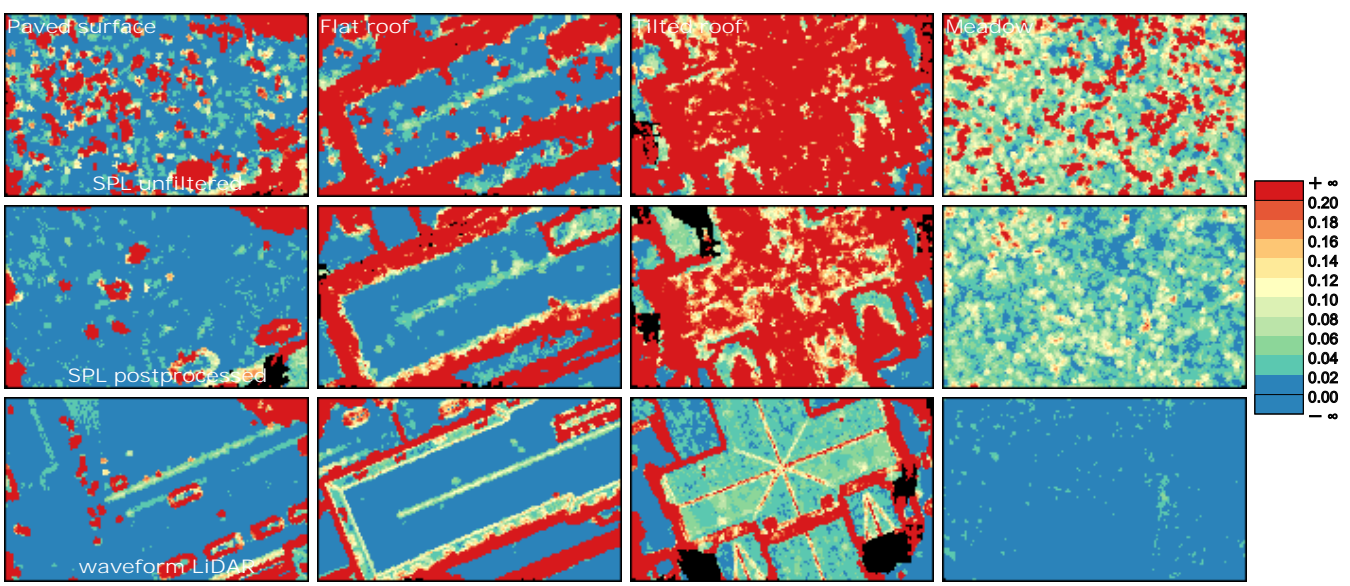

Figure 9. Color coded point cloud dispersion maps (std.dev. [m]) for selected surface patches (extent: 40x24 $\mathrm{m}^{2}$ ); upper row: SPL unfiltered, middle row: SPL postprocessed, lower row: waveform LiDAR

one of the two overlapping flight strips. However, both datasets feature a full coverage at street level with a flight block point density in the range of 25 points $/ \mathrm{m}^{2}$. The red and black low-density areas in the SPL dataset (cf. Figure 7c) mainly occur in the inner courtyards beyond the building perimeter caused by scan shadows of either the buildings themselves or trees. The high-density areas at the building edges (facades) are less pronounced in the SPL dataset. As stated above, this might also be a result of point cloud postprocessing, which tends to eliminate points in the vegetation as well as on the facades.

As an additional standard product of ALS quality control, the relative height differences between overlapping flight strips were calculated and analyzed. Figure 8 shows the respective results for both test areas and data acquisitions. Large deviations of more than $10 \mathrm{~cm}$, indicated as dark blue/red color tones in Figure $8 \mathrm{a}$ and $b$, are to be expected in vegetated areas when scanning semi transparent objects from different viewing directions. Therefore, only smooth surface parts are displayed for the city test area. The white color tones in the DEM of Difference map of the waveform LiDAR dataset (Figure 8c) indicate a good fit, which is further confirmed by the histogram of the height residuals shown in Figure $8 \mathrm{e}$ (median: $0 \mathrm{~cm}$, std.dev.: $2 \mathrm{~cm}$ ). The corresponding DEM of Difference map of the SPL dataset is dominated by light color tones with a small positive bias (median: $+1 \mathrm{~cm}$ ). With a standard deviation of $6 \mathrm{~cm}$, the dispersion is three times higher compared to the waveform LiDAR dataset, but still within the range specified by the manufacturer. The moderate precision drop is also well in line with comparable studies in the literature (Mandlburger and Jutzi, 2018).

Finally, the local dispersion of the 3D point clouds at smooth surface parts was analyzed in more detail for selected regions featuring (i) horizontal paved surfaces, (ii) tilted roofs, (iii) flat roofs, and (iv) open grassland. It is noted that only data from a single flight strip were used in order to separate point cloud dispersion from strip fitting precision. The results are shown in Figure 9 for the unfiltered and postprocessed SPL dataset (upper/middle row) and the waveform LiDAR point clouds, respectively (lower row). In addition, Table 1 summarizes the median dispersion of all smooth grid posts (i.e., std.dev. $<20 \mathrm{~cm}$ ). While the dispersion is generally lower for the waveform LiDAR point cloud 
compared to SPL, the drop of precision varies for individual test surfaces. Horizontal surfaces (paved road, flat roof) exhibit a low dispersion in the $\mathrm{cm}$ range for both acquisition methods, with SPL being only marginally less precise than waveform LiDAR (drop factor about 1.5). Steeply tilted surfaces show higher dispersion values (waveform LiDAR: $3.1 \mathrm{~cm}$ ), and the SPL precision loss factor goes up to about 3 . For the meadow test patch, finally, the waveform LiDAR dispersion is in the $\mathrm{cm}$ range. SPL is about 5-times less precise here, but still better than $10 \mathrm{~cm}$, the vertical accuracy value reported in the SPL100 data sheet (Leica, 2019). It is noted that the precision measures for the meadow section need to be treated with caution as the different acquisition dates might have an influence on the results.

\begin{tabular}{lcccc}
\hline data type & paved surface & flat roof & tilted roof & meadow \\
\hline SPL unfiltered & 1.5 & 1.2 & 9.8 & 5.8 \\
SPL postproc. & 1.1 & 1.0 & 9.5 & 3.9 \\
waveform LiDAR & 1.0 & 0.7 & 3.1 & 1.0 \\
\hline
\end{tabular}

Table 1. Point cloud dispersion $[\mathrm{cm}]$

\section{CONCLUSIONS AND OUTLOOK}

This article described the basic principles and specific properties of conventional full waveform laser scanning and of the single photon based technologies Geiger-mode LiDAR and Single Photon LiDAR, respectively. The main focus was on SPL, as a respective sensor (Leica Geosystems SPL100) is commercially available meanwhile, also outside the U.S. Compared to conventional linear-mode LiDAR, SPL features a higher performance for large area topographic mapping while maintaining comparable point densities of typically 8 points $/ \mathrm{m}^{2}$.

In 2018, the city administration of Vienna commissioned a test flight with the SPL100 sensor covering approx. $30 \%$ of the city area of Vienna including the adjacent forest area. The data were captured in July 2018. In a case study, the SPL point clouds were evaluated and compared to an independent data acquisition of the same area in September 2018 with a conventional FWF laser scanner (RIEGL, VQ-1560i). In summary, it can be stated that (i) SPL requires less flight strips to cover the same area due to the higher flying altitude and the resulting broader swath width, (ii) the waveform LiDAR 3D point clouds provide a sharper and more concise mapping of both topography and buildings, (iii) SPL exhibits vegetation penetration capabilities even under leaf-on conditions, but waveform LiDAR provided a better ground point coverage, (iv) the conical scan mechanism of the SPL100 is well suited for capturing building facades, but a high strip overlap is necessary to provide side looks from different viewing directions, (v) postprocessing of the unfiltered SPL point clouds reduced the dispersion of neighboring points but also tended to eliminate usable points on facades and within the vegetation canopy, and (vi) the local point cloud dispersion is generally lower for waveform LiDAR by a factor of 1-5 depending on the surface type, but still satisfactory dispersion values below $10 \mathrm{~cm}$ were obtained for SPL.

In the present article, we highlighted the pros and cons of the respective technologies, both of which are evolving. During data analysis specific questions surfaced, which can only be answered by future investigations. If highest accuracy is required, full waveform based multi-photon LiDAR is still the technology of first choice, but if the product specification tolerates a precision level in the $\mathrm{dm}$ range, Single Photon LiDAR offers a better area coverage performance.

\section{References}

Agishev, R., Comerón, A., Bach, J., Rodriguez, A., Sicard, M., Riu, J. and Royo, S., 2013. Lidar with SiPM: Some capabilities and limitations in real environment. Optics and Laser Technology 49, pp. 86-90.

Briese, C., Pfennigbauer, M., Lehner, H., Ullrich, A., Wagner, W. and Pfeifer, N., 2012. Radiometric calibration of multiwavelength airborne laser scanning data. In: ISPRS Annals of the Photogrammetry, Remote Sensing and Spatial Information Sciences, Vol. 1, pp. 335-340.

Clifton, W. E., Steele, B., Nelson, G., Truscott, A., Itzler, M. and Entwistle, M., 2015. Medium altitude airborne geiger-mode mapping lidar system. In: Proc. SPIE, Vol. 9465, pp. $9465-$ $9465-8$.

Degnan, J., 2002. Photon-counting multikilohertz microlaser altimeters for airborne and spaceborne topographic measurements. Journal of Geodynamics 34(3-4), pp. 503-549.

Degnan, J. J., 2016. Scanning, Multibeam, Single Photon Lidars for Rapid, Large Scale, High Resolution, Topographic and Bathymetric Mapping. Remote Sensing 8(11), pp. 923-958.

Harris, 2019. Website of Harris Geiger-mode LiDAR. https://www.harris.com/solution/geiger-mode-lidar (last accessed: 2019-01-11.

Kim, S., Lee, I. and Kwon, Y. J., 2013. Simulation of a geigermode imaging LADAR system for performance assessment. Sensors (Switzerland) 13(7), pp. 8461-8489.

Lehner, H. and Briese, C., 2010. Radiometric calibration of full-waveform airborne laser scanning data based on natural surfaces. In: International Archives of Photogrammetry, Remote Sensing and Spatial Information Sciences, XXXVIII/7B, pp. 360-365.

Mandlburger, G. and Jutzi, B., 2018. Feasibility Investigation on Single Photon Lidar Based Water Surface Mapping. ISPRS Annals of Photogrammetry, Remote Sensing \& Spatial Information Sciences IV(October), pp. 10-12.

Leica, 2019. Leica SPL100 single photon LiDAR sensor data sheet. https://leica-geosystems.com/products/airbornesystems/topographic-lidar-sensors/leica-spl100.

Pfennigbauer, M., Wolf, C., Weinkopf, J. and Ullrich, A., 2014. Online waveform processing for demanding target situations. In: Proc. SPIE, p. $90800 \mathrm{~J}$.

Romano, M. E., 2015. Geiger Mode LiDAR. In: Proceedings of the ASPRS IGTF Conference 2015

Shan, J. and Toth, C. K. (eds), 2018. Topographic Laser Ranging and Scanning: Principles and Processing. 2 edn, CRC Press, Boca Raton, FL.

Stoker, J. M., Abdullah, Q. A., Nayegandhi, A. and Winehouse, J., 2016. Evaluation of Single Photon and Geiger Mode Lidar for the 3D Elevation Program. Remote Sensing 8(9), pp. 716767.

Sugarbaker, L. J., Constance, E. W., Heidemann, H. K., Jason, A. L., Lukas, V., Saghy, D. L. and Stoker, J. M., 2014. The 3D Elevation Program Initiative A Call for Action. USGS Circular 1399.

Swatantran, A., Tang, H., Barrett, T., DeCola, P. and Dubayah, R., 2016. Rapid, High-Resolution Forest Structure and Terrain Mapping over Large Areas using Single Photon Lidar. Scientific Reports.

Ullrich, A. and Pfennigbauer, M., 2016. Linear LIDAR versus Geiger-mode LIDAR: impact on data properties and data quality. In: Proc. SPIE, Vol. 9832, pp. 983204-983217.

Ullrich, A. and Pfennigbauer, M., 2018. Noisy lidar point clouds: impact on information extraction in high-precision lidar surveying. In: Proc.SPIE, Vol. 10636, pp. 10636 - 10636 - 6.

Vosselman, G. and Maas, H.-G. (eds), 2010. Airborne and Terrestrial Laser Scanning. Whittles Publishing, UK.

Wagner, W., 2010. Radiometric calibration of small-footprint full-waveform airborne laser scanner measurements: Basic physical concepts. ISPRS Journal of Photogrammetry and Remote Sensing 65(6), pp. 505-513. 